\title{
Differential patterns of dynamic cardiovascular regulation as a function of task ${ }^{1}$
}

\author{
Barry E. Hurwitz *, Richard A. Nelesen, Patrice G. Saab, Joachim H. Nagel, \\ Susan B. Spitzer, Marc D. Gellman, Philip M. McCabe, Donna J. Phillips \\ and Neil Schneiderman \\ Behavioral Medicine Research Center, Departments of Psychology, Medicine, and Biomedical \\ Engineering, University of Miami, P.O. Box 248185, Coral Gables, FL 33124, USA
}

\begin{abstract}
In cardiovascular reactivity studies, interpretations of the processes supporting the blood pressure response may become problematic when systolic blood pressure, diastolic blood pressure, and heart rate all increase in response to a behavioral challenge. Therefore, in addition to evaluating these cardiovascular responses, this study examined cardiac output, total peripheral resistance and systolic time intervals derived from impedance cardiogram, electrocardiogram and phonocardiogram recordings during a speech stressor, a mirror tracing task, and a foot cold pressor test. All of the behavioral stressors elicited increases in blood pressure and heart rate, with the largest changes occurring during the overt speech. Based on the examination of the response patterns of the underlying hemodynamic variables it would appear that, in both men and women, the blood pressure increase during the speech preparation period was supported by increased cardiac output; the speech itself resulted in a mixed pattern of increased cardiac output and total peripheral resistance; whereas, the mirror tracing and cold pressor tasks produced increased total peripheral resistance. Although men and women produced similar response patterns to the behavioral challenges, sex differences in the estimates of myocardial contractility were observed during rest. These results provide evidence that different behavioral stressors can produce a distinct yet integrated pattern of responses, whose differences may be revealed, when impedance cardiography is used, to derive sufficient response measures for assessing dynamic cardiovascular processes.
\end{abstract}

Keywords: Acute stressurs; Impedance cardiography; Blood pressure; Cardiac output; Total peripheral resistance; Systolic time intervals

\section{Introduction}

Selye (1956) postulated that stress was a response of physiological arousal that could be induced by many stimuli. The evoked response, however, was thought to follow a specific physiological pattern and as such would be the

* Corresponding author.

This work was supported by Research Grant HL36588 and Research Training Grant HL07426 from the National Heart, Lung, Blood Institute of Health. 
same regardless of the eliciting event. 'The notion of non-specific response has been challenged by researchers who noted that certain stimulus attributes give rise to characteristically different patterns and magnitudes of cardiovascular response (see Mason, 1975). This understanding is apparent in the psychophysiological strategy that has been used to study the relationship between physiological responses to behavioral challenges and cardiovascular disease processes. This strategy involves presenting laboratory stressors and measuring the stressor-related changes in various physiological response measures. The underlying assumption is that exposure to behavioral stressors leads to incrcases in sympathetic rcactivity and that this in turn may be important in studying disease processes. Considerable attention in this research has focused on the magnitude of reactivity to behavioral stressors as a marker or even a possible contributing factor in the pathogenesis of cardiovascular disease (e.g. Krantz \& Manuck, 1984). Commonly, in studies of cardiovascular reactivity, increases in sympathetic reactivity have been inferred from increases in blood pressure, heart rate (HR) and sometimes catecholamine responses (e.g. Tischenkel, Saab, Schneiderman, Nelesen, Pasin, Goldstein, Spitzer, Woo-Ming \& Weidler, 1990). Recently several laboratories have employed other measures, such as systolic time intervals, as estimates of sympathetic input to the myocardium (e.g. Sherwood, Allen, Obrist \& Langer, 1986; Allen, Obrist, Sherwood \& Crowell, 1987; Sung, Lovallo, Teague, Pincomb \& Wilson, 1989; Kelsey \& Guethlein, 1990; Saab, Llabre, Hurwitz, Frame, Reineke, Fins, McCalla, Cieply \& Schneiderman, 1992). Most of these studies have used impedance cardiography to assess hemodynamic and electromechanical aspects of cardiovascular functioning. This methodology is a relatively unohtrusive, non-invasive technique for measuring stroke volume (SV), cardiac output $(\dot{Q})$, total peripheral resistance (TPR) and systolic time intervals (see review Sherwood, Allen, Fahrenberg, Kelsey, Lovallo \& Van Doornen, 1990). By assessing the pattern of alterations in these cardiovascular variables it is possible to obtain an understanding of the underlying mechanisms for the regulation of the blood pressure response to behavioral challenge.

The central nervous system appears to be organized to produce integrated patterns of response rather than isolated alterations in single variables (Hilton, 1975). It is clear from the human and animal literature that two major sympathetic patterns are associated with behavioral responses to stress (see review Schneiderman, 1976; Schneiderman \& McCabe, 1989). Pattern 1, described in relation to the defense reaction or active coping, has been characterized by increases in systolic blood pressure (SBP) and $\dot{Q}$ with no increase in TPR; whereas, Pattern 2, described in relation to aversive vigilance or inhibitory coping, has been characterized by increases in diastolic blood pressure (DBP), SBP, and TPR. The magnitude and specificity of components of the physiological response pattern may be influenced by 
stimulus attributes and behavioral demands of the stressors. Therefore, in addition to the two basic patterns described above, a mixed pattern may occur as a function of the behavioral task itself and its context or differences in individual history, perceptual and response styles and other similar variables (Schneiderman \& McCabe, 1989).

Several recent studies have begun to assess the extent to which situational stereotypy (Lacey, 1967) exists in behavioral tasks as a function of $\dot{Q}$ and TPR. Although these studies have suggested that behavioral stressors may differ from one another in the cardiovascular response patterns that they elicit (e.g. Allen, Obrist, Sherwood \& Crowell, 1987; Allen \& Crowell, 1989; Sherwood, Dolan \& Light, 1990), these studies have used tasks that induce a Pattern 1 response profile (increases in $\dot{Q}$ ) or mixed response profile (increases in $\dot{Q}$ and TPR). In preliminary research conducted during the past few years, we have observed that mirror tracing (a psychological task) and the cold pressor test (a physical and psychological task) both elicit responses that are consistent with Pattern 2. The primary purpose of the present paper was to assess in both men and women the patterns of changes in $\dot{Q}$, TPR, and systolic time interval estimates of contractility, as well as blood pressure and HR elicited by these tasks with those of a speech stressor task. The speech task produces relatively large SBP and HR increases, and hence was expected to elicit the Pattern 1 response.

Most of the reactivity studies assessing both men and women have used only blood pressure and HR to characterize the cardiovascular response, with inconsistent agreement. Several studies have observed that compared with women, men are more responsive with blood pressure and less responsive with HR to behavioral challenge (Baker, Dearhorn, Hastings, \& Hamberger, 1984; Lane, White \& Williams, 1984; Stoney, Davis \& Matthews, 1987; Stoney, Matthews, McDonald \& Johnson, 1988, Mathews, Davis, Stoney, Owens \& Caggiula, 1991). Results in other studies examining blood pressure and HR reactivity have found no sex differences (Jorgenson \& Houston, 1981; Manuck, Craft \& Gold, 1978; Van Doornen, 1986). One study reported that women were more blood pressure and HR reactive than men in four of six stressors (Stone, Dembroski, Costa, \& MacDougall, 1990). However, in another comparison of sex differences in reactivity to two mental arithmetic and two speaking stressors, no differences in blood pressure reactivity were observed (Girdler, Turner, Sherwood, \& Light, 1990). Although the latter study, using impedance cardiography to derive $\dot{Q}$ and TPR, did observe greater $\dot{Q}$ and less TPR reactivity in women than men, this occurred in only one of the four tasks. Thus the literature appears inconclusive regarding sex differences in cardiovascular response to behavioral challenge. This may in part be a consequence of the relative paucity of studies that examine in men and women the underlying hemodynamic response patterns supporting blood pressure and HR reactivity. 
Therefore, the primary purpose of this study was to determine whether different behavioral challenges (i.e. mirror tracing, cold pressor and speech) can produce distinct cardiovascular response patterns. A secondary purpose of study was to determine if the response patterns elicited by these tasks were similar in both men and women, since previous studies have shown that at least on some tasks men and women apparently differ.

\section{Method}

\section{Subjects}

Thirty white, native born, US citizens were recruited through local newspaper advertising and were paid for their participation in this study. The subjects were equally represented by sex and included 15 women and 15 men. There were significant sex differences between men and women in height $(t(28)=6.06, P<0.001)$ and weight $(t(28)=3.46, P<0.002)$, but not in age, or body mass index. Mean $( \pm \mathrm{SE})$ height was $161.5( \pm 3) \mathrm{cm}$ for women and $180.1( \pm 2) \mathrm{cm}$ for men. Body weight was $66.8( \pm 4) \mathrm{kg}$ for women and 85.2 $( \pm 3) \mathrm{kg}$ for men. The age range was 25-54 years, with a mean age of 39.1 $( \pm 3)$ years for the women, and $38.9( \pm 3)$ years for the men. Body mass index was $25.2( \pm 0.02) \mathrm{kg} \mathrm{m}^{-2}$ for the women and $26.3( \pm 0.01) \mathrm{kg} \mathrm{m}^{-2}$ for the men.

Prior to the testing session, all subjects, in a medical history assessment, reported the absence of any cardiopulmonary or other medical disorders. This was confirmed by physical examination, fasting blood chemistry analysis and 12 lead electrocardiogram (ECG). Eligibility for participation required that the subject be free of medication, report no history or show no evidence of dizzy spells, angina pectoris, ventricular arrhythmias, myocardial infarction, cerebrovascular disease, seizure disorders, diabetes or severe asthma. Only subjects with blood pressure in the normotensive range (i.e., DBP $<90$ $\mathrm{mm} \mathrm{Hg}$ ) were included in this study. Mercury sphygmomanometer blood pressure measures were performed on two separate days prior to the testing day to determine the blood pressure status for each subject. There were no significant sex differences in these blood pressure measures. The mean casual blood pressure averaged over 2 days was $120( \pm 4) / 76( \pm 3) \mathrm{mm} \mathrm{Hg}$ for men and $117( \pm 4) / 72( \pm 2) \mathrm{mm} \mathrm{Hg}$ for women.

Within the female sample, all subjects were pre-menopausal, three subjects were taking oral contraceptives at the time of participation in the study; six women had a family history of one parent with hypertension and two women had a family history of both parents with hypertension. Within the male sample, four men had a history of one parent with hypertension and 
one man had a history of both parents with hypertension. One woman and three men smoked cigarettes.

\section{Physiological recording apparatus}

Measurements of blood pressure were obtained using a Critikon Dinamap (model 1846SX) Adult/Pediatric Vital Signs Monitor. The occlusion cuff was placed on the upper portion of the arm. The ECG, phonocardiogram (PCG), and impedance cardiogram (ICG) signals were recorded with a Grass polygraph. The ECG lead configuration was selected to enhance the $Q$ and $T$ waves for the purpose of facilitating off-line signal event detection. The three ECG electrodes were placed on either side of the rib cage and on the sternum, inferior to the suprasternal notch. Either a Lead I or II configuration was selected on the basis of the magnitude of the $Q$ wave. One active ECG lead was placed in either V4, V5, or V6 position so that a maximal $T$-wave amplitude was obtained, which optimizes computer detection of the end of the $T$-wave. From the PCG the first and second heart sounds were recorded by placing a phonotransducer (Hewlett Packard-21050A) on the cardiac window in the second or fourth intracostal space just to the left of the sternum.

The ICG was derived using a tetrapolar aluminum and mylar tape (No. T-8001, Instrumentation for Medicine,) electrode configuration. Of the four ICG leads, Lead II was affixed first in a location just superior to the suprasternal notch of the thorax, at the base of the neck. Lead I was placed precisely $3 \mathrm{~cm}$ superior to Lead II on the upper neck. Lead III was placed around the thorax overlaying the xiphoid process at the inferior end of the sternum. Lead IV was placed on the lower portion of the thorax precisely 5 $\mathrm{cm}$ inferior to Lead III. These four ICG leads were placed $180^{\circ}$ around the body ${ }^{2}$, while the subject was standing erect, such that the impedance electrodes were parallel to the floor. This was done to ensure uniform current spread over the body. An alternating current of $4 \mathrm{~mA}$ at $100 \mathrm{kHz}$ from the Minnesota Impedance Cardiograph (Model 304B) was passed through the outer electrodes and the signals were recorded from the inner

2 The half hand impedance lead configuration has been favorably compared with invasive techniques of SV determination (Watanabe, Kamide, Torii \& Ochiai, 1981). It also has been recommended in the committee report on impedance cardiography as one possible lead configuration that may be used (Sherwood, Allen et al., 1990). Since that time we have come to learn from preliminary data collected in this laboratory that the shorter electrode bands produce a larger mean thoracic impedance by about $3 \mathrm{Ohms}$ than the full $\left(360^{\circ}\right)$ electrode bands. This has the effect of artificially reducing the derived SV values. However, since the present study was designed to consider within-subjects the patterns of changes in the ICG-derived parameters, the reduction in level of SV values should not impair the comparisons relevant to the experimental hypotheses. 
two clectrodes. The two signals derived from the ICG signal were the mean thoracic impedance $\left(Z_{\mathrm{o}}\right)$ and the first derivative of the pulsatile change in thoracic impedance $(\mathrm{d} Z / \mathrm{d} t)$.

The ECG and PCG signals were relayed from the polygraph to an A/D converter (DT 2801) sampling at $1 \mathrm{kHz}$ per channel for $30 \mathrm{~s}$ and stored in an IBM PC-AT computer. The $\mathrm{d} Z / \mathrm{d} t$ signal was relayed directly from the impedance cardiograph to the A/D converter and similarly sampled and stored in the computer so that the impedance signal would be free of the attenuating effects imposed by polygraphic high frequency filtering. Impedance $\mathrm{d} Z / \mathrm{d} t$ and $Z_{\mathrm{o}}$ calibration signals were also stored for later conversion of the $\mathrm{d} Z / \mathrm{d} t$ signal to Ohms $\mathrm{s}^{-1}$ and $\mathrm{SV}$ derivation.

The 30-s samples of ECG, PCG, and $\mathrm{d} Z / \mathrm{d} t$ were ensemble averaged off-line by a computer program which summed the digitized beat-by-beat waveforms, time synchronized to the ECG $R$-wave, and divided by the number of cardiac cycles. The ensemble average was then graphically displayed and the waveform events were scored by computer signal processing techniques described previously (Nagel, Shyu, Reddy, Hurwitz, McCabe \& Schneiderman, 1989; Hurwitz, Shyu, Reddy, Schneiderman \& Nagel, 1990). Each ensemble average including the placement of cursors over the computer-detected events was then viewed by the operator. The program permitted the operator to correct interactively any events scored incorrectly by the computer. The seven signal events detected by the signal processing program were: (a) the ECG $Q$-wave onset, representing the onset of electrical systole; (b) the ECG $R$-wave, corresponding to the peak of electrical ventricular depolarization; (c) the end of the ECG $T$-wave, representing the end of ventricular repolarization; (d) the onset of the second heart sound (S2) from the PCG signalling the end of left ventricular ejection and closure of the aortic valve; the $\mathrm{S} 2$ was used only to aid in verifying that the $\mathrm{d} Z / \mathrm{d} t X$-wave was properly identified; (e) the $\mathrm{d} Z / \mathrm{d} t B$-wave, representing the onset of left ventricular ejection; (f) $\mathrm{d} Z / \mathrm{d} t_{\max }$, the peak of the change in thoracic impedance, representing the maximal left ventricular ejection velocity; $(\mathrm{g})$ the $\mathrm{d} Z / \mathrm{d} t X$-wave onset ${ }^{3}$, which corresponds in time with $\mathrm{S} 2$ onset, representing the completion of left ventricular ejection. The precision of the computer software for detecting these signal events has been described previously

${ }^{3}$ The present study used the $X$-wave onset rather than the more commonly used $X$-wave minimum to denote the left ventricular ejection endpoint or aortic valve closure. Specifically, the aortic valve closure has commonly been demarcated by the onset of the second heart sound of the PCG (Lewis, Rittgers, Forester \& Boudoulas, 1977). In a previous study it has been shown, during resting and behavioral challenge conditions, that compared with the second heart sound onset the $X$-wave onset occurs more proximally in time (3-5 ms) than the $X$-wave minimum (21-25 ms) (Nagel et al., 1990). Therefore, to avoid an overestimation of LVET and SV, the $X$-wave onset was used. 
(Nagel, Shyu, Reddy, Hurwitz, McCabe \& Schneiderman, 1989). If the operator observed any artifact superimposed upon the ICG waveform, which might be present as a result of respiration, limb, torso or electrode movement, the operator could exercise the option of rejecting the contaminated cardiac cycles and repeating the ensemble average construction. Artifact contamination occurred in less than $2 \%$ of the ensemble averages. None of the reconstructed ensemble averages, used less than $70 \%$ of the original number of cardiac cycles.

\section{Derivation of indices of cardiovascular performance}

Several indices of cardiovascular performance were derived from the ensemble average of the computer samples of ECG, PCG, and ICG. The quantitative measurements and calculations were as follows: (a) interbeat interval was determined on a beat-by-beat basis by measuring the interval (ms) between the peaks of two successive ECG $R$-waves; (b) HR, in beats per minute (bpm), was derived from the mean interbeat interval per 30-s ensemble average; (c) left ventricular ejection time (LVET) was derived by measuring the interval (ms) between the $B$-wave local minimum and the onset of the $X$-wave of the $\mathrm{d} Z / \mathrm{d} t$; (d) the $Q Z$ interval (ms) is the interval between the ECG $Q$-wave onset and the peak of the $\mathrm{d} Z / \mathrm{d} t$ wave; (e) the $B Z$ interval $(\mathrm{ms})$ is the interval between the minimum in the $B$-wave and the peak of the $\mathrm{d} Z / \mathrm{d} t$ wave; (f) the ejection velocity $\left(\mathrm{d} Z / \mathrm{d} t_{\max }\right.$ ) is measured in Ohms $\mathrm{s}^{-1}$ and was calculated as the difference in amplitude between the minimum in the $B$-wave and the peak amplitude of the $\mathrm{d} Z / \mathrm{d} t$ signal; $(\mathrm{g})$ Heather Index (HI) is measured in Ohms $\mathrm{s}^{-1}$ and is defined as the ratio of $\mathrm{d} Z / \mathrm{d} t_{\max }$ to the $Q Z$ interval; (h) Acceleration Index (ACI) reflects the acceleration of the rapid ventricular ejection phase following aortic opening, measured in Ohms $\mathrm{s}^{-1}$ and is defined as the ratio of $\mathrm{d} Z / \mathrm{d} t_{\max }$ to the $B Z$ interval; the $\mathrm{HI}$ and ACI have been suggested to reflect left ventricular contractility (Heather, 1969; Kizakevich, Teague, Jochem, Nissman, Niclou \& Sharma, 1989); (i) SV was calculated using the Kubicek formula (Kubicek, Witsoe, Patterson, \& From, 1969), $\mathrm{SV}=$ rho $*\left(L / Z_{\mathrm{o}}\right)^{2} * \mathrm{LVET}^{*} \mathrm{~d} Z / \mathrm{d} t_{\max }$. Rho is the blood resistivity in Ohms $\mathrm{cm}^{-1}$ and was calculated using the formula, rho = $53.2 \mathrm{e}^{(0.022) \mathrm{HCT}}$, where HCT is the hematocrit as measured from the collected blood samples (Kubicek, Witsoe, Patterson \& From, 1969). The closest HCT in time with the ensemble average period was used to calculate rho. The electrode distance $(L)$ in $\mathrm{cm}$ between ICG Leads II and III was measured while the subject was seated in the chair prior to the first baseline period; (j) $\dot{Q}$, measured in liters per minute was calculated by multiplying the HR by $\mathrm{SV}$; (k) TPR measured in peripheral resistance units was derived by dividing the ratio of mean arterial pressure $/ \dot{Q}$ by the constant 16.67 (Guyton, 1981; Sherwood, Allen, Fahrenberg, Kelsey, Lovallo \& Van Dooren, 1990). The 
mean arterial pressure and $\dot{Q}$ measures derived temporally from the closest blood pressure reading and 30 -s sample were used to calculate TPR.

\section{Procedures}

During a preliminary session informed consent, height and weight, a fasting blood sample and resting mercury blood pressures were obtained. Subjects were instructed to eat a light meal prior to the testing session, but were reminded to refrain from smoking (for $2 \mathrm{~h}$ ), and were instructed not to consume coffee (for $18 \mathrm{~h}$ ), tea, cocoa, or caffeinated soft drinks (for $6 \mathrm{~h}$ ) prior to their appointment.

On the testing day, between 09:00 and 10:00 h, a heparinized butterfly needle (Kowarski-Cormed thromboresistant $18 \mathrm{ga}$. blood withdrawal needle and tubing set) was inserted into an antecubital vein and attached to a small $(10 \mathrm{~cm} \times 9 \mathrm{~cm})$ portable peristaltic pump (Cormed ML6). Physiological measures (blood pressure, ECG, PCG and ICG) were recorded while subjects were at rest or engaged in behavioral tasks. The subjects were seated in a padded chair in a room adjacent to the control room and observed through a one-way mirror. There were three behavioral challenge periods, speech, mirror tracing, and cold pressor, that were presented to the subjects in this fixed order. These tasks were preceded by a pre-task baseline period respectively lasting $20 \mathrm{~min}, 30 \mathrm{~min}$ and $20 \mathrm{~min}$. The additional $10 \mathrm{~min}$ of inter-task interval following the first task was provided because preliminary studies indicated that time for blood pressure recovery may be more prolonged after completion of the speech task than following the other tasks. Baseline data were collected during the $3 \mathrm{~min}$ prior to the end of each baseline period.

\section{Speech stressor}

The speech task consisted of two phases, a 3-min speech preparation period which led directly into a 3-min speech talking period (Saab, Matthews, Stoney, \& McDonald, 1989). Subjects were individually instructed to prepare a speech in which they are told that they were stopped at a local department store and accused of stealing a belt that they had actually purchased there the previous week. The subjects were asked to explain to the store manager why they were detained erroneously. Subjects were told that the video camera in front of them was taping their talk and that they would be evaluated by expert raters on their performance and appearance. During the 3 -min preparation phase the subjects were provided with a card that listed five points to be addressed and instructed to prepare the speech until cued to begin speaking. The 3-min speaking period began when a red light on the video camera was turned on. If the subjects stopped talking before the end of the time period they were reminded to continue, if only to summarize and reiterate the main points of their talk. 


\section{Mirror tracing}

The mirror tracing task required the subjects to trace the outline of a star by using its reflection in an adjacent mirror for $3 \mathrm{~min}$. The image as viewed in the mirror is normal in the vertical plane, but reversed in the horizontal plane and hence presents the subject with a visual-motor integration problem. In addition, at 30 -s intervals an experimenter who was standing beside the subject made critical statements about the subject's performance. ${ }^{4}$

\section{Cold pressor}

Subjects placed their left foot into a bucket of icc water (half ice, half water, with temperature about $4^{\circ} \mathrm{C}$ ) for $90 \mathrm{~s}$. Instructions were to keep their foot in the water until told to remove it.

\section{Sampling of blood pressure, indices of cardiovascular performance, and blood}

During the last $3 \mathrm{~min}$ of the pre-task baseline periods blood pressure was sampled twice at $1.5 \mathrm{~min}$ intervals. During the $3 \mathrm{~min}$ of speech preparation, speech talking and mirror tracing tasks blood pressure measurement was initiated at the 15-s and 2-min time points. During the cold pressor task, blood pressure was also sampled twice, at 0- and 45-s time points. ECG, ICG, and PCG were collected during baseline and task periods for 30-s samples initiated within 10-15 s of blood pressure initiation. Blood was collected for hematocrit determination as integrated 3-min samples during the three baseline periods, speech preparation, speech talk and mirror tracing tasks, and for the $1.5 \mathrm{~min}$ of cold pressor task.

\section{Results}

There were two measures of each physiological parameter for each baseline and task period. Since there were no significant differences between repeated measures within each baseline period on any of the measures, physiological parameters were averaged over the pre-task baseline period. The task measures were averaged, as well, to form an overall mean for each

${ }^{4}$ Debriefing of the subjects followed the task by about 6 min and consisted of informing the subjects about the uniformity of these comments for all subjects, about the intent of the verbal challenges, and that no further comments would be made about their task performance. The critical comments and verbal challenges performed by the experimenter were included in this procedure to introduce potential anger-inducing properties, which have been shown to correlate with cardiovascular responsiveness (Suarez \& Williams, 1990). In a subsequent study in our laboratory, which used the mirror tracing task without the experimenter comments, the pattern of response did not differ from those reported in the present study (Saab et al., 1992). 
Table 1

Mean $( \pm S E)$ values for cardiovascular measures averaged across the three baseline periods

\begin{tabular}{lccl}
\hline Measure $^{\mathrm{a}}$ & \multicolumn{1}{l}{ Men } & Women & $P^{\mathrm{b}}$ \\
\hline SBP (mmHg) & $119.0 \pm 4.2$ & $110.1 \pm 2.9$ & NS \\
DBP (mmHg) & $70.2 \pm 2.7$ & $67.4 \pm 2.1$ & NS \\
HR (beats min & \\
SV $(\mathrm{ml})$ & $69.3 \pm 3.1$ & $72.8 \pm 1.9$ & NS \\
$\dot{Q}\left(\mathrm{I} \mathrm{min}^{-1}\right)$ & $65.8 \pm 8.8$ & $57.5 \pm 5.0$ & NS \\
TPR (units) & $4.4 \pm 0.5$ & $4.2 \pm 0.5$ & NS \\
HI $\left(\Omega \mathrm{s}^{-2}\right)$ & $1.42 \pm 0.16$ & $1.45 \pm 0.17$ & NS \\
ACI $\left(\Omega \mathrm{s}^{-2}\right)$ & $10.2 \pm 0.8$ & $15.3 \pm 1.0$ & $p<0.001$ \\
$\mathrm{~d} Z / \mathrm{d} t\left(\Omega \mathrm{s}^{-2}\right)$ & $25.3 \pm 2.8$ & $44.0 \pm 4.4$ & $p<0.005$ \\
$Z_{\mathrm{o}}(\Omega)$ & $1.83 \pm 0.13$ & $2.72 \pm 0.16$ & $p<0.001$ \\
\hline
\end{tabular}

a See text for definitions of measures.

${ }^{b}$ Probability of significance of the statistical comparison between genders.

behavioral challenge. All statistical analyses were performed using the SPSS-x statistical package.

Pre-task baseline levels

Initially, differences were assessed in the physiological variables during the three pre-task baseline periods as a function of sex. To do this a $2(\operatorname{sex}) \times 3$ (pre-task baseline period) analysis was performed using a multivariate analysis of variance (MANOVA) approach to a repeated measures analysis for each dependent variable separately. The analysis for each cardiovascular measure revealed no significant change across the baseline periods. Mean \pm SE values for the dependent measures averaged across the three baselines are presented in Table 1. Significant sex main effects were found for the two contractility indices, $\mathrm{HI}(F(1,28)=17.73, P<0.001)$ and $\mathrm{ACI}(F(1,28)=$ $12.83, P<0.005)$. The greater $\mathrm{HI}$ and $\mathrm{ACI}$ values for women were a result of larger $\mathrm{d} Z / \mathrm{d} t_{\max }$ in women than men $(F(1,28)=19.79, P<0.001)$. However, there was a trend approaching significance for shorter $B Z$ interval in women than men $(F(1,28)=3.31, P<0.08)$, reflecting more rapid ventricular ejection velocity, with respective mean values of $66.8( \pm 4.4)$ and $77.5( \pm 3.8) \mathrm{ms}$. The difference in $\mathrm{HI}$ and $\mathrm{ACI}$ between men and women is so large it seems unlikely to reflect solely contractility differences. Alternatively, the shortened $B Z$ interval in females may be a result of differences in the conduction of ICG current. This may be related to sex differences in thoracic anatomy, reflected by $Z_{\mathrm{n}}$. Women had greater $Z_{\mathrm{o}}$ than men $(F(1,28)=6.50, P<0.05)$. When the HI is recalculated by correcting $\mathrm{d} Z / \mathrm{d} t_{\max }$ for $Z_{\mathrm{o}}$, the ejection velocity index (EVI) is derived in units per second (Kelsey \& Guethlein, 1990). When the EVI was calculated, sex differences during baseline re- 
mained $(F(1,28)-5.38, P<0.05)$, indicating that sex differences in $Z_{\mathrm{o}}$ do not account for the sex differences in myocardial contractility. Respective EVI mean values for men and women were $0.408( \pm 0.04)$ and $0.545(+0.05)$ units $\mathrm{s}^{-1}$. There were no other significant sex differences found during the baseline periods. 5

Although there was a significant interaction between sex and baseline period for $\dot{Q}(F(2,27)=4.50, P<0.05)$, this was accounted for by a similar significant interaction for $\operatorname{SV}(F(2,27)-3.88, P<0.05)$, without any significant alteration in HR across the baseline periods. The SV interaction was due to a trend for a small $\mathrm{SV}$ decrease for men (respective mean ml values over baselines were $67.4 \pm 9.4,66.7 \pm 8.6,63.4 \pm 8.7$ ) in contrast with a small $\mathrm{SV}$ increase for women (respective mean $\mathrm{ml}$ values over baselines were $56.3 \pm 6.2,57.9 \pm 5.8,58.2 \pm 6.2$ ) across the three baseline periods.

\section{Response to behavioral challenge}

Univariate ANOVA procedurcs were performed in the following analyses to assess the response to the behavioral challenges. (As each dependent variable was analysed separately and since these analyses used only two repeated measures, MANOVA tests of significance were inappropriate as univariate tests are equivalent to MANOVA under these circumstances.) These ANOVAs used sex as the between-subject variable and examined for each physiological variable independently whether changes in reactivity occurred from pre-task baseline to task. Thus, the speech task analyses compared baseline vs. speech preparation, and compared speech preparation vs. speech delivery. The mirror tracing and cold pressor tasks were analysed similarly (baseline vs. task).

Figures 1,2 and 3 depict the mean change from baseline, for men and women, for SBP, DBP, HR, $\dot{Q}^{6}$, TPR, $\mathrm{SV}^{6}, \mathrm{HI}$ and $\mathrm{ACI}$ during speech preparation, speech talk, mirror tracing and cold pressor tasks.

\section{Speech preparation}

The preparation for the delivery of the speech produced significant elevations in SBP $(F(1,28)=58.07, P<0.001)$ and DBP $(F(1,28)=60.97$, $P<0.001)$ relative to baseline. Although no significant change in SV oc-

${ }^{5}$ Commonly in the literature sex differences in baseline SBP and HR have been observed (e.g. Matthews et al., 1991). Based upon a power analysis using the present data it should be possible to detect baseline sex differences for these variables with a much larger sample size. With a power of 0.80 and $\alpha=0.05$, a significant difference for SBP and HR would be found if 34 and 114 subjects per group, respectively, were used.

${ }^{6}$ When the SV and $\dot{Q}$ data were corrected for body surface area to derive stroke index and cardiac index and analyzed accordingly, no differences were found between the pattern of results obtained with SV and stroke index and the results obtained with $\dot{Q}$ and cardiac index. 

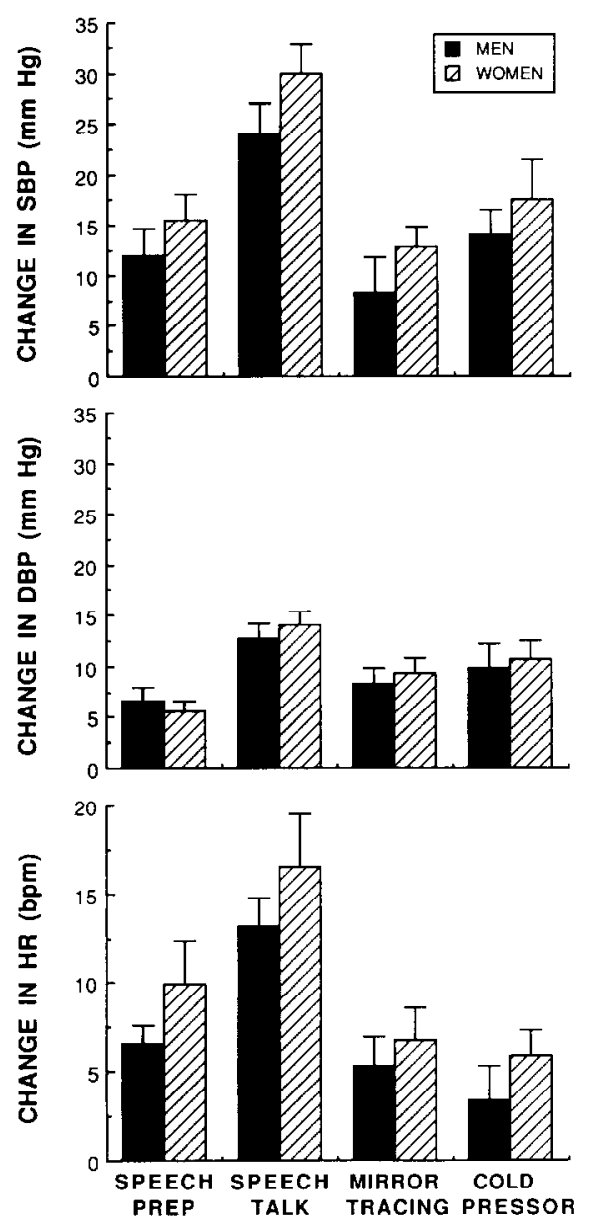

Fig. 1. Mean $( \pm S E)$ change of cardiovascular measures from baseline during speech preparation, speech talk, mirror tracing, and cold pressor: systolic blood pressure (SBP); diastolic blood pressure (DBP); and heart rate (HR).

curred, there was a significant increase in $\operatorname{HR}(F(1,28)=39.52, P<.001)$. This resulted in a significant increase in $\dot{Q}(F(1,28)=7.87, P<0.01)$, without any change in TPR. Both estimates of sympathetic myocardial tone, HI $(F(1,28)=6.99, P<0.05)$ and ACI $(F(1,28)=5.55, P<0.05)$ significantly increased from baseline during this period. The main effect for sex observed during baseline were maintained for $\mathrm{HI}(F(1,28)=9.76, P<0.005)$ and ACI $(F(1,28)=11.08, P<0.005)$ during this period. There were no significant interactions of sex with response from pre-task to speech preparation. 

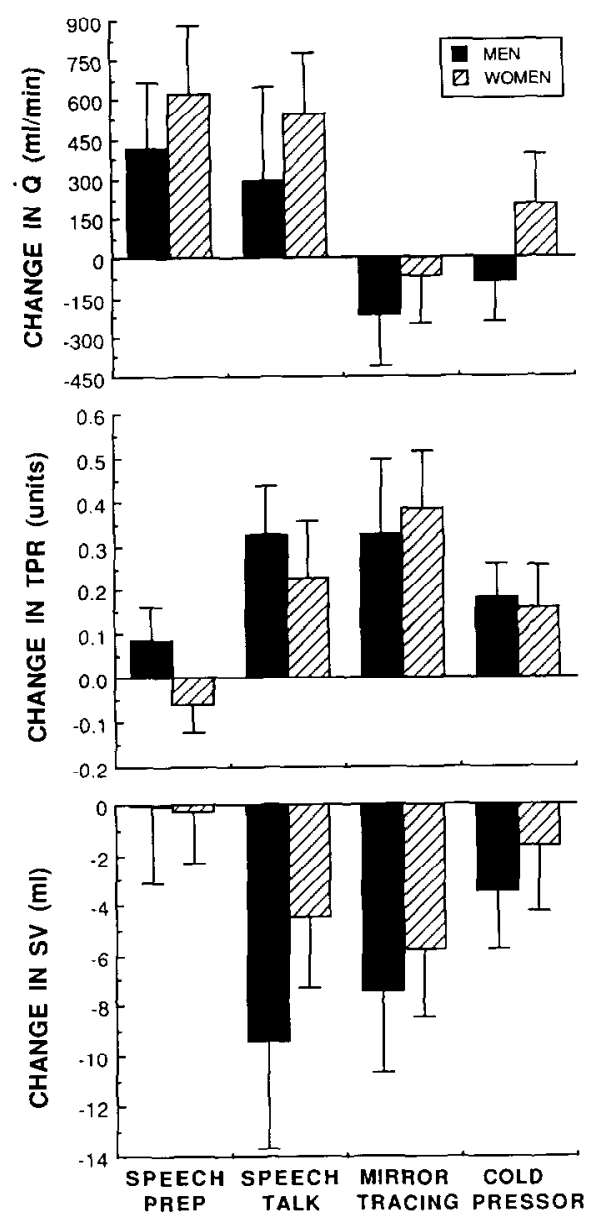

Fig. 2. Mcan ( $\perp S E$ ) change of cardiovascular measurcs from bascline during specch preparation, speech talk, mirror tracing, and cold pressor: cardiac output $(\dot{Q})$; total peripheral resistance (TPR) and stroke volume (SV).

\section{Speech delivery}

The speaking phase of the task produced a further significant increase in SBP $(F(1,28)=60.34, P<0.001)$ and DBP $(F(1,28)=56.75, P<0.001)$ relative to the preceding preparation period. HR also significantly increased, $(F(1,28)=34.75, P<0.001)$, but SV decreased, $(F(1,28)=11.54, P<0.005)$. Consequently, there was no change in $\dot{Q}$ from the speech preparation phase. There was, however, a significant increase from the preparation phase in TPR, $(F(1,28)=21.51, P<0.001)$. The HI remained significantly increased $(F(1,28)=4.35, P<0.05)$ relative to baseline and did not differ from the 


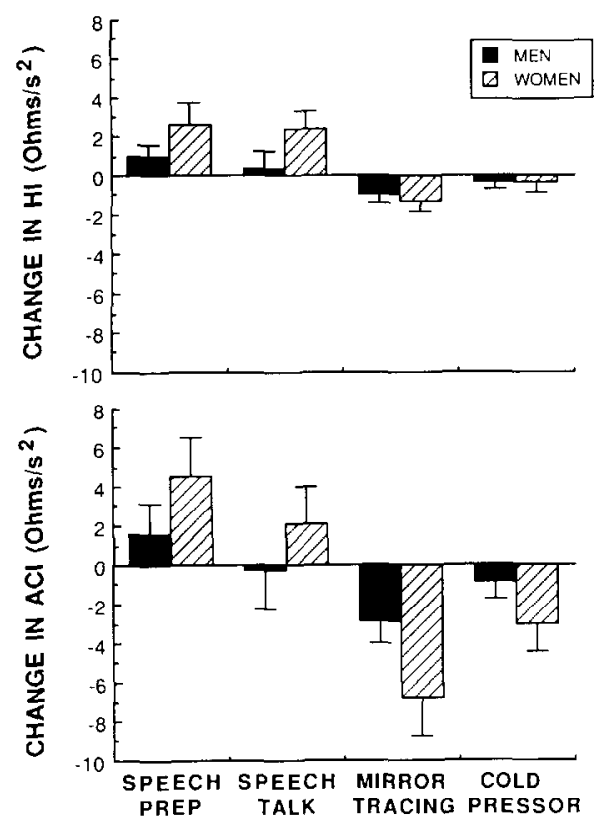

Fig. 3. Mean $( \pm \mathrm{SE})$ change in systolic time interval ratios from baseline during speech preparation, speech talk, mirror tracing, and cold pressor: Heather Index (HI) and Acceleration Index $(\mathrm{ACl})$.

preceding preparation period; however, a significant decline in ACI from speech preparation to talk $(F(1,28)=5.26, P<0.05)$ to pre-task level was observed. The sex main effect in $\mathrm{HI}(F(1,28)=9.54, P<0.005)$ and ACI $(F(1,28)=11.66, P<0.005)$ was also maintained during this period. There were no significant interactions of sex with response from speech preparation to talking.

\section{Mirror tracing task}

The mirror tracing produced elevations in both $\operatorname{SBP}(F(1,28)=27.20$, $P<0.001)$ and DBP $(F(1,28)=62.63, P<0.001)$ from pre-task baseline levels. HR significantly accelerated, $(F(1,28)=25.69, P<0.001)$, but SV significantly decreased $(F(1,28)=9.77, P<0.005)$. The result of these countering influences was that no $\dot{Q}$ change from baseline occurred. In contrast, the rise in blood pressure was accompanied by a significant increase in TPR $(F(1,28)=11.05, P<0.005)$. Both HI and ACI significantly decreased during mirror tracing $(\mathrm{HI},(F(1,28)=13.21, P<0.001$; ACI $(F(1$, $28)=17.17, P<0.001))$. The sex main effect for $\mathrm{HI}(F(1,28)=16.23, P<$ $0.001)$ and $\mathrm{ACI}(F(1,28)=12.72, P<0.001)$ continued during this stressor. There were no significant interactions of sex with task response relative to baseline. 


\section{Cold pressor task}

The cold foot immersion elicited significant increases in $\operatorname{SBP}(F(1,28)=$ 46.19, $P<0.001)$ and DBP $(F(1,28)=45.55, P<0.001)$ relative to pre-task baseline levels. A small significant increase in $\operatorname{HR}$ occurred $(F(1,28)=15.04$, $P<0.005)$, but no change in SV was observed. $\dot{Q}$ did not significantly change from baseline, but a significant increase in TPR $(F(1,29)=7.54, P<0.01)$ occurred. Although no significant $\mathrm{HI}$ change from baseline was found during the cold pressor task, ACI significantly decreased during this period ( $F$ (1, $28)=5.70, P<0.05)$. The sex main effect remained for $\mathrm{HI}(F(1,28)=20.82$, $P<0.001)$ and $\mathrm{ACI}(F(1,28)=13.98, P<0.001)$ during this challenge. No significant interactions of sex with response to task relative to baseline emerged.

\section{Between-task comparisons of response magnitude}

Change scores for physiological variables during each task were computed for each subject by subtracting the pre-task baseline mean from the given task mean. A $2(\mathrm{sex}) \times 4$ (speech preparation vs. speech talk vs. mirror tracing vs. cold pressor) analysis was performed using a MANOVA approach to a repeated measures analysis for each dependent variable separately to determine whether the four task periods differed in the magnitude of change from pre-task levels. The distinct pattern of responses to each of the behavioral challenges was similar in both men and women. There were no significant main effects of sex nor any significant interactions of sex and task period observed. Estimates based upon the power analyses (Kraemer \& Thiemann, 1987) of the change scores for each of the four task periods (power $=0.80$ and $\alpha=0.05$ ) indicated that even if the sample size per sex group was tripled no sex differences would have resulted for any of the variables reported herein. The MANOVA analyses revealed significant main effects among tasks for all of the following variables: $\operatorname{SBP}(F(3,27)=30.44$, $P<0.001)$, DBP $(F(3,27)=20.21, P<0.001)$, HR $(F(3,27)=16.39, P<$ $0.001)$, SV $(F(3,27)=5.39, P<0.005), \dot{Q}(\mathrm{~F}(3,27)=11.74, P<0.001)$, TPR $(\mathrm{F}(3,27)=14.79, P<0.001), \mathrm{HI}(\mathrm{F}(3,27)=8.36, P<0.001)$ and $\mathrm{ACI}(\mathrm{F}(3$, 27) $=6.13, P<0.005)$.

Subsequent paired analyses used the Bonferonni correction procedure for multiple comparisons as recommended (Keselman \& Keselman, 1988). These follow-up comparisons (all $P$ 's $<0.0083$ ) revealed that the SBP elevation during the talking phase of the speech task was significantly greater than the SBP increases during each of the other behavioral challenges, which did not differ in SBP elevation. The speech talking phase also produced the greatest increase in DBP, compared with the mirror tracing and cold pressor tasks; whereas, speech preparation produced less DBP increase than the other tasks. 
The HR accelerations induced by the tasks were differentiated by a greater response during the speech delivery than the speech preparation; the speech preparation response was not different from the increase in the mirror tracing task, but was greater than the acceleration observed during the cold pressor task. There were no differences between the negative SV changes induced during the speech talk and mirror tracing tasks. These tasks produced lower SV changes than the speech preparation and cold pressor tasks, which did not differ in level of SV. The increases in $\dot{Q}$ observed during the speech preparation and talk did not differ, but were greater than the other two tasks. In contrast, speech talking, mirror tracing and cold pressor displayed equivalent increases in TPR, which exceeded the TPR observed during the speech preparation task.

Between task comparisons for HI and ACI changes revealed the same pattern of results. Specifically, the speech preparation period induced changes in these systolic time interval ratios that were not different from the speech talking values, but were significantly larger than cold pressor alterations. The $\mathrm{HI}$ and ACI during the cold pressor, however, was significantly greater than the decreased levels induced by the mirror tracing task.

The speech talking period was distinguished from the other tasks in that a greater magnitude of SBP, DBP and HR was observed (see Figs. 1 and 2). SV was maintained during speech preparation and talking, but decreased during the other two stressors. Thus, the speech task was the only behavioral challenge where a significant increase in $\dot{Q}$ was observed, with $\dot{Q}$ increasing during speech preparation to a level that was maintained during the talking phase. It was the speech preparation phase which also induced the largest increases in contractility estimated by the $\mathrm{HI}$ and $\mathrm{ACI}$ ratios (see Fig. 3). In contrast, with the exception of speech preparation period, all of the other tasks elicited an increase in TPR (see Fig. 2), with respective increases relative to baseline of 25,25 and $12 \%$ for speech talk, mirror tracing and cold pressor tasks.

\section{Discussion}

The present study used impedance cardiography to derive measures of $\dot{Q}$, TPR and estimates of contractility ( $\mathrm{HI}$ and $\mathrm{ACI}$ ) in addition to assessments of blood pressure and HR to examine two major issues: (1) do the three behavioral challenges elicit different cardiovascular response patterns? and (2) do the response patterns differ as a function of gender? The behavioral challenges could be discriminated by different patterns of change in the measures of cardiovascular function. Substantial increases in blood pressure occurred during each stressor. Although the speaking phase of the speech task produced the largest SBP increase and the DBP response was less when 
preparing for the speech, the tasks were otherwise not systematically differentiated by the blood pressure response magnitude. During the two distinct periods of the speech task, two corresponding patterns of response were observed. The blood pressure elevation in the first period, speech preparation, occurred as a consequence of an increase in $\dot{Q}$. The second period, the talking phase, produced even greater blood pressure elevations, which appeared to result from both myocardial and vascular influences, i.e. sustained increases in $\dot{Q}$ coupled with an increase in TPR. In contrast, the mirror tracing and cold pressor tasks displayed blood pressure elevations that could be solely accounted for by elevations in TPR.

The pattern of $\mathrm{HI}$ and $\mathrm{ACI}$ alterations to the behavioral challenges have been suggested to reflect changes in myocardial contractility (Heather, 1969; Kizakevich, Teague, Jochem, Nissman, Niclou \& Sharma, 1989). It should be noted, however, that the ACI reflects myocardial performance during the rapid ventricular ejection phase following aortic opening, whereas the $\mathrm{HI}$ reflects myocardial performance during both pre-ejection and ejection phases of the cardiac cycle. During speech preparation HI and ACI increased, which is consistent with the suggestion that this task elicited increases in myocardial contractility. During the speech talking phase, the elevated levels of $\mathrm{HI}$ appeared to decline, but did not change significantly, indicating that the enhanced myocardial contractility was continued throughout this task period. Although the significant decline in ACI suggests that contractility may have decreased from previously elevated levels during this period, or that perhaps the ACI measure may be differentially influenced by the increasing afterload occurring during the speaking challenge. In the other two tasks, mirror tracing and cold pressor, the $\mathrm{HI}$ ratio exhibited a decrease and no change in the mirror tracing and cold pressor tasks respectively; whereas the ACI decreased in both of these periods, suggesting that there was, at least, no increase in myocardial contractility evoked by these two stressors.

The greater HI, ACI and EVI in the women during baseline, supports the possibility of an underlying sex difference in myocardial contractility. Evidence from the animal literature suggests that structural and functional sex differences exist in rats. Females have greater heart weight per body weight and greater aortic flow than males (Berg \& Harmison, 1955; Shreiner, Weisfeldt \& Shock, 1969). Moreover, in vitro studies of intrinsic myocardial contractile performance have revealed that females exhibit greater velocity of fiber shortening and shorter time to peak tension than males, even though no sex differences were observed in myocardial elasticity or in the peak tension developed (Capasso, Remily, Smith, \& Sonnenblick, 1983). This evidence suggests that sex-related differences in myocardial contractile function may exist.

The focus in a large proportion of the sex-related reactivity literature has been on whether men and women may differ in the magnitude of blood 
pressurc or HR rcactivity to certain stressors, with inconclusive findings. In contrast, the present study examined the pattern of hemodynamic response in men and women to various behavioral challenges and clearly demonstrated the pronounced similarity in response profile produced by men and women to the behavioral challenges. In a recent study, also of men and women, women tended to display greater $\dot{Q}$ responses while men tended to respond with greater alterations in TPR (Girdler, Turner, Sherwood \& Light, 1990). However, it should be noted that these sex differences in $\dot{Q}$ responsiveness were present only in a speech task in which the subjects verbally recalled a previously examined text. In the two mental arithmetic tasks and in an evaluative speech task similar to that used herein, men and women responded similarly, which is in agreement with the findings of the present study. Power analyses of the present study indicated that with much greater numbers of subjects per gender group (i.e. ranging from 50 to 126), magnitudinal differences might be observed in single cardiovascular responses (i.e. SBP, HR, HI and ACI) during certain stressors. However, the significance of finding isolated differences in response magnitude pales when presented with independent groups in which the entire pattern of cardiovascular responses differs, reflecting an entirely different underlying hemodynamic response mechanism. For example, while the white men and women herein displayed similar response profiles, in a recent study on an entirely different sample, which compared the cardiovascular response of white men with black men to similar stressors used in this study, the black men demonstrated a response stereotypy in which the blood pressure elevations were predominantly mediated by increased TPR irrespective of the behavioral challenge (Saab, Llabre, Hurwitz, Frame, Reineke, Fins, McCalla, Cieply \& Schneiderman, 1992). Therefore, other subject variables such as ethnicity may interact with gender and may be important to understanding the similarities and differences in cardiovascular regulation related to gender.

In summary, based on the findings of blood pressure, HR, $\dot{Q}$, TPR and contractility estimates during the behavioral challenges, different integrated cardiovascular response profiles could be discriminated. The response to the preparation phase of the speech task resembled the Pattern 1 profile, in which the elevated blood pressure was supported by increased $\dot{Q}$ and myocardial contractility; whereas a mixed pattern including increased $\dot{Q}$ and TPR and increased, although declining, myocardial contractility appears to have occurred when the subjects were engaged in the talking phase of the speech task. The mirror tracing and cold pressor tasks evoked a Pattern 2 response profile including increases in blood pressure that were accompanied by increases in TPR with no change in $\dot{Q}$ and no increase in myocardial contractility from resting level. Future pharmacologic studies could reveal the relative $\beta$ - and $\alpha$-adrenergic contribution to the Pattern 1 and 2 response profiles. 
The use of a research strategy, which uses a single or a limited number of tasks and response measures to characterize cardiovascular system response underestimates the complexity of the central neural blood pressure regulation. Behavioral challenges, with varying psychological and physical aspects, can induce varying alterations in $\alpha$ - and $\beta$-adrenergic activity and sympathetic/parasympathetic balance and hence can have differing, although specifiable, effects on the sympathetic and cardiovascular systems (e.g. Dimsdale \& Moss, 1980; Halter, Stratton \& Pfeifer, 1984; Sherwood, Allen, Obrist \& Langer, 1986). We have shown that when techniques such as impedance cardiography are employed to derive a comprehensive cardiovascular assessment selected behavioral challenges produce distinct integrated patterns of cardiovascular response. Therefore, the present study emphasizes the need to: (1) employ strategically the behavioral challenges, with an emphasis on the underlying physiological mechanisms mediating the response; and (2) examine sufficient response measures for the hemodynamic and electromechanical properties of the cardiovascular response patterns and their variations to be identified.

\section{References}

Allen, M.T. \& Crowell, M.D. (1989). Patterns of autonomic response during laboratory stressors. Psychophysiolngy, 26, 603-614.

Allen, M.T., Obrist, P.A., Sherwood, A. \& Crowell, M.D. (1987). Evaluation of myocardial and peripheral vascular responses during reaction time, mental arithmetic and cold pressor tasks. Psychophysiology, 24, 648-656.

Baker, L.J., Dearborn, M., Hastings, J.E. \& Hamberger, K. (1984). Type A behavior in women: a review. Health Psychology, 3, 477.

Berg, B.N. \& Harmison, C.R. (1955). Blood pressure and heart size in aging rats. Journal of Gerontology, 10, 416-419.

Capasso, J.M., Remily, R.M., Smith, R.H. \& Sonnenblick, E.H. (1983). Sex differences in myocardial contractility in the rat. Basic Research in Cardiology, 78, 156-171.

Dimsdale, J.E. \& Moss, J. (1980). Plasma catecholamines in stress and exercise, Journal of the American Medical Association, 243, 340-342.

Girdler, S.S., Turner, J.R., Sherwood, A. \& Light, K.C. (1990). Gender differences in blood pressure control during a variety of behavioral stressors. Psychosomatic Medicine, 52, 571-591.

Guyton, A.C. (1981). Textbook of medical physiology. Philadelphia: W.B. Saunders Co.

Halter, J.B., Stratton, J.R. \& Pfeifer, M.A. (1984). Plasma catecholamines and hemodynamic responses to stress states in man. Acta Physiologica Scandinavica, 527, 31-38.

Hcathcr, L.W. (1969). A comparison of cardiac output values by the impedance cardiograph and dye dilution techniques in cardiac patients. In W.G. Kubicek, D.A. Witsoe \& R.P. Patterson (Eds.), Development and evaluation of an impedance cardiographic system to measure cardiac output and other cardiac parameters (pp. 247-258). Houston, IX, NASA Publication CR 101965.

Hilton, S.M. (1975). Ways of viewing the central nervous control of the circulation-old and new. Brain, 87, 213-219. 
Hurwitz, B.E., Shyu, L.-Y., Rcddy, S.P., Schnciderman, N. \& Nagel, J.II. (1990). Coherent ensemble averaging techniques for impedance cardiography. In H.T. Nagle \& J.N. Brown (Eds.), Computer-based medical systems (pp. 228-235). IEEE Computer Society Press, Washington.

Jorgenson, R.S. \& Houston, B.K. (1981). Family history of hypertension, personality patterns, and cardiovascular reactivity to stress. Psychosomatic Medicine, 48, 102-117.

Kelsey, R.M. \& Guethlein, W. (1990). An evaluation of the ensemble averaged impedance cardiogram. Psychophysiology, 27, 24-33.

Keselman, H.J. \& Keselman, J.C. (1988). Comparing repeated measures means in factorial designs. Psychophysiology, 25, 612-618.

Kizakevich, P.N., Teague, S.M., Jochem, W.J., Nissman, D.B., Niclou, R. \& Sharma, M.K. (1989). Detection of ischemic response during treadmill exercise by computer-aided impedance cardiography. Proceedings of the 2nd annual IEEE symposium on computer-based medical systems, IEEE catalog No. 89CH2755-7, 10-15.

Kraemer H.C. \& Thiemann, S. (1987). How many subjects? Statisical power analysis in Research. Newbury Park: Sage Publications, Inc.

Krantz, D.S. \& Manuck, S.B.(1984). Acute psychophysiologic reactivity and risk of cardiovascular disease: a review and methodologic critique. Psychological Bulletin, 96, 435-464.

Kubicek, W.G., Witsoe, D.A., Patterson, R.P. \& From, A.H.L. (1969). Development and evaluation of an impedance cadiographic system to measure cardiac output and other cardiac parameters (NASA-CR-101965). Houston: National Aeronautics and Space Administration.

Lacey, J.I. (1967). Somatic response patterning and stress: Some revisions of activation theory. In M.H. Appley \& R. Trumble (Fds.), Psycholngical stress: Issues in Research (pp. 14-42). New York: Appleton-Century-Crofts.

Lane, J.D., White, A.D. \& Williams, R.B. (1984). Cardiovascular effects of mental arithmetic in Typc A and Typc B females. Psychophysiology, 21, 39-46.

Lewis, R.P., Rittgers, S.E., Forester, W.F. \& Boudoulas, H. (1977). A critical review of systolic time intervals. Circulation, 56, 146-158.

Manuck, S.B., Craft, S. \& Gold, K.J. (1978). Coronary-prone behavior pattern and cardiovascular response. Psychophysiology, 15, 403-411.

Mason, J.W. (1975). A historical view of the stress field: Part II. Journal of Human Stress, 1, 22-36.

Matthews, K.A., Davis, M.C., Stoney, C.M., Owens, J.F. \& Caggiula, A.R. (1991). Does the gender relevance of the stressor influence sex differences in psychophysiological responses? Health Psychology, 10, 112-120.

Nagel, J.H., Shyu, L.Y., Reddy, S.P., Hurwitz, B.E., McCabe, P.M. \& Schneiderman, N. (1989). New signal processing techniques for improved precision of noninvasive impedance cardiog raphy. Annals of Biomedical Engineering, 17, 517-534.

Saab, P.G., Llabre, M.M., Hurwitz, B.E., Frame, C.A., Reineke, L.J., Fins, A.I., McCalla, J., Cieply, L.K. \& Schneiderman, N. (1992). Myocardial and peripheral vascular responses to behavioral challenges and their stability in black and white Americans. Psychophysiology, 29, 384-397.

Saab, P.G., Matthews, K.A., Stoney, C.M. \& McDonald, R.H. (1989). Premenopausal and post-menopausal women differ in their cardiovascular and neuroendocrine responses to behavioral stressors. Psychophysiology, 26, 270-280.

Schneiderman, N. (1976). Animal models relating behavioral stress and cardiovascular pathology. In T. Dembroski, S.M. Weiss, J.L. Shields, S.G. Haynes \& M. Feinleib (Eds.), Coronary-prone behavior (pp. 155-182). New York: Springer.

Schneiderman, N. \& McCabe, P.M. (1989). Psychophysiologic strategies in laboratory research. In N. Schneiderman, S.M. Weiss \& P.G. Kaufman (Eds.), Handbook of research methods in cardiovascular behavioral medicine (pp.349-364). New York: Plenum. 
Selye, H. (1956). The Stress of Life. McGraw-Hill. New York.

Sherwood, A., Allen, M.T., Fahrenberg, J., Kelsey, R.M., Lovallo, W.R. \& van Doornen, L.J.P. (1990). Methodological guidelines for impedance cardiography. Psychophysiology, 27, 1-23.

Sherwood, A., Allen, M.T., Obrist, P.A. \& Langer, A.W. (1986). Evaluation of beta-adrenergic influences on cardiovascular and metabolic adjustments to physical and psychological stress. Psychophysiology, 23, 89-104.

Sherwood, A., Dolan, C.A. \& Light, K.C. (1990). Hemodynamics of blood pressure responses during active and passive coping. Psychophysiology, 27, 656-668.

Shreiner, D.P., Weisfeldt, M.L. \& Shoch, N.W. (1969). Effects of age, sex, and breeding status on the rat heart. American Journal of Physiology, 217, 176-180.

Stone, S.V., Dembroski, T.M., Costa, Jr., P.T. \& MacDougall, J.M. (1990). Gender differences in cardiovascular reactivity. Journal of Behavioral Medicine, 13, 137-156.

Stoney, C.M., Matthews, K.A., McDonald, R.H. \& Johnson, C.A. (1988). Sex differences in lipid, lipoprotein, cardiovascular, and neuroendocrine responses to acute stress. Psychophysiology, $25,645-656$

Stoney, C.M., Davis, M.C. \& Matthews, K.A. (1987). Sex differences in physiological response to stress and coronary heart disease: A causal link. Psychophysiology, 24, 127-131.

Suarez, E.C. \& Williams, R.B., Jr. (1990). The relationships between dimensions of hostility and cardiovascular reactivity as a function of task characteristics. Psychosomatic Medicine, 52, $558-570$.

Sung, B.H., Lovallo, W.R., Teague, S.M., Pincomb, G.A. \& Wilson, M.F. (1989). Noninvasive measures of left ventricular mass and resting hemodynamics in borderline hypertension. Psychophysiology, 26, S59.

Tischenkel, N.J., Saab, P.G., Schneiderman, N., Nelesen, R.A., Pasin, R.D., Goldstein, D.A., Spitzer, S.B., Woo-Ming, R. \& Weidler, D.J. (1990). Cardiovascular and neurohumoral responses to behavioral challenge as a function of race and sex. Health Psychology, 8, 503-524.

Van Doornen, L.J.P. (1986). Sex differences in physiological reactions to real life stress and their relationship to psychological variables. Psychophysiology, 23, 657-662.

Watanabe, T., Kamide, T., Torii, Y. \& Ochiai, M. (1981). A convenient measurement of cardiac output by half-taped impedance cardiography. Japanese Journal of Medical Electronics and Biological Engineering, 19, 30-34. 